\title{
ADDRESSING DIVERSITY AND GENDER ISSUES IN A CORNER- STONE DESIGN COURSE
}

\author{
Sarah R. Nicholson, W. Patrick Neumann, Mary F. (Frankie) Stewart, and Filippo A. Salustri \\ Ryerson University \\ Corresponding Author: salustri@,ryerson.ca
}

\begin{abstract}
In the cornerstone engineering design course for Mechanical and Industrial Engineering undergraduates at Ryerson University, students' design approaches were being negatively affected by gender and other biases. Therefore, the course was modified to encourage students to explore these biases, with an initial emphasis on gender so that they may design with a fuller sense of women's issues. This novel endeavour aimed to change the course's culture via awareness, and by connecting equity, diversity, and inclusion to an engineering context. Qualitative analysis of student reports before and after these modifications showed that the intervention led to user groups that more closely matched actual demographics and included a higher number of women, LGBTQ, and elderly Personas than before. Furthermore, the qualitative descriptions showed less of a skewed tendency to attribute positive characteristics to men and negative characteristics to women after the course modifications were implemented. Student surveys indicated that there was a potential cultural shift within the course, and a broadening of student focus to include equity, diversity, and inclusion when undertaking an engineering design project.
\end{abstract}

Keywords: Inclusive Design, Diversity, Gender Issues, Engineering

\section{INTRODUCTION}

The instructors and teaching assistants of MEC325 (Introduction to Engineering Design), the cornerstone engineering design course for Mechanical and Industrial Engineering undergraduates at Ryerson University, have noticed that students' design approaches were limited by a lack of inclusion and a presence of gender biases. In response, the authors have undertaken to modify the course to encourage students to explore these biases, with an emphasis on gender issues, so that their designs more appropriately serve diverse populations. Our goal is to change the course's culture by bringing awareness to these issues, and discussing them within an engineering context, in order to provide more opportunities for traditionally underrepresented students to engage more fully with design, to improve communication skills, and for all students to gain a more nuanced sense of equity, diversity, and inclusion in engineering design.

\section{PROJECT DESIGN AND EXECUTION}

\subsection{Overview}

MEC 325 is the $3^{\text {rd }}$ semester cornerstone design course taken by all Mechanical and Industrial Engineering students at Ryerson University. A key component of the course is a semester-long, team-based design project. Teams must submit a formal written report at the end of the semester discussing their design and how they developed it. The lectures and tutorials in the course explain the design process and provide oversight by TAs and instructors. The course is typically taught by Neumann and Salustri.

Since MEC325 is intended to also introduce Human Factors, the focus of the course is on product design; that is, the design of things that humans will use directly.

Our design philosophy is that people have limitations, but it is engineering designers who, by the design choices they make, create disability in users who cannot meet the demands of a designed product. In short, engineers create limitations on users, and our goal is to teach students to try to minimize those potential exclusions.

A key aspect of our approach is to begin the design process by identifying Personas - hypothetical archetypal users that represent typical actual users - and designing products that satisfy the needs of those Personas.

More information about MEC325 is available [5, 6].

The authors secured funding from Ryerson's Learning and Teaching Office to support a recent course TA (Nicholson) to carry out the research and development of courseware interventions as described below.

The project was designed to include three stages.

Stage 1: In Summer 2018, the interventions were designed by Nicholson, in collaboration with Neumann, Stewart, and Salustri. To design the interventions, Nicholson analyzed a sample of student design reports from Fall 2017.

Stage 2: In Fall 2018, the interventions were deployed in MEC 325. 
Stage 3: In Winter 2019, a follow-up analysis was conducted by Salustri to determine the impact of the interventions.

Each stage is described further below.

\subsection{Stage 1: Intervention Development}

Research conducted during Summer 2018 included a review of a sample of student project reports from Fall 2017, when Nicholson was a TA for the course. The sample included all the reports from two sections of the class (11 student teams and 189 Personas total). The design project brief, and the instructors, were the same for all teams in the sample. The review included (a) an analysis of the demographics of the Personas that students developed, including age groups, gender, family role, and occupation; and (b) a qualitative analysis using informal coding of key terms that suggested gender or diversity biases in students. Furthermore, Nicholson gathered background information and teaching examples from an interdisciplinary breadth of literature, and consulted with local experts at Ryerson University, including faculty members, and staff at the Learning and Teaching Office and the Equity and Community Inclusion Office.

As a result of these analyses, we identified the following issues:

- over-representation of men, and underrepresentation of women, among Personas;

- incorrect and negative stereotypical characterizations of abilities of women and the elderly; and

- $\quad$ incorrect, naïve, and negative stereotypical characterization of the degree of agency and influence of women and the elderly.

It was not therefore surprising that the designs that students developed in 2017 were commonly assessed by the instructors as insufficient for their target users, particularly with respect to economic, safety, and social implications. The lack of diversity, equity, and inclusion in the reports was leading to incomplete designs which had the potential to exclude underrepresented groups.

The authors then collaboratively reviewed possible courseware interventions that could be implemented under the given time and resource constraints, to be deployed by Fall 2018. Nicholson subsequently developed:

- a new design project brief for a baby stroller, intended to lead students to focus on designing for women by using an artifact more commonly thought of as a "women's issue";

- new slides for studios and lectures on accessible design, how to design for women, inclusive design, and engineers in society;

- lists of resources that students could use to learn about demographic issues and inclusive design strategies;
- guidelines for teaching assistants to encourage thinking about diversity;

- modifications to rubrics to account for diversity; and

- $\quad$ an exit survey for students to provide feedback on the new courseware.

Particular attention was given to courseware supporting the generation of appropriately diverse Personas and their appropriate use throughout the downstream stages of designing, especially in requirements specification and in concept evaluation.

Simultaneous with Nicholson's work, Salustri developed a new tool for Persona representation. In previous years, including Fall 2017, students were told to describe each Persona with up to 200 words of free-form text per Persona. Over the years, we had learned that this format was extremely difficult to grade and allowed for too much variability. Even with several worked examples covered carefully in lecture, students had a very difficult time developing relevant Personas, and graders had a very difficult time assessing the free-form text.

Therefore, in Fall 2018, we deployed a chart structure for Persona specification (see [6] for details). We reasoned that the chart would provide more guidance to students to define Personas more relevant to the design brief, and would be easier to grade.

\subsection{Stage 2: Deployment}

In Fall 2018, the instructors deployed the material developed by Nicholson.

The new baby stroller design brief was used in two sections of the class (approximately 50 students).

Slide packs were made available as reading assignments, with key slides being discussed in lecture and referred to as appropriate in tutorials. The slide packs covered the development of Personas, accessibility in design, designing for women, inclusive design, and engineering within the context of a diverse society.

Due to the size of the class (typically between 280 and 300 students), the instructors depend on TAs to be the "first line of defence" to help students. Because of this, a special guide for TAs was distributed at the beginning of the term. This guide provided a summary of identified problems in MEC325, background information on gender and diversity issues, techniques that TAs could apply to recognize biases in student work and to help students address those biases.

The rubrics used to assess milestone and final reports were visible to students. Time was taken in lecture to point out those rubric elements that related to gender and diversity, to emphasize their significance in measuring design quality. 
At the end of the semester, a voluntary and anonymous exit survey was administered to assess student opinion of the gender and diversity materials used.

\subsection{Stage 3: Post-Intervention Analysis}

In Winter 2019, Salustri repeated Nicholson's analysis on a sample of Fall 2018 design reports. Nicholson was consulted regularly to ensure that the analysis was performed consistently between the two years. Two sections (10 student teams and 188 Personas total) were used as the sample. The same design project brief was given to both sections, and it was the same brief as was used in 2017. A different TA was assigned to these two sections in 2018 than was done in 2017, but the newer TA was very well suited to support the new courseware. In this way, the instructors hoped to lessen the sources of variation between the 2017 and 2018 reports.

\section{SUMMARY OF RESULTS}

This section summarizes the results of the analyses conducted on the two sample sets, from 2017 and 2018.

\subsection{Quantitative Analyses}

The quantitative analyses were demographic in nature. Characteristics measured were: number of male and female Personas generated, the number of "mother" and "father" Personas, and their ages. Baseline data was taken from the Statistics Canada database. The gender of each persona was determined from the report's explicit descriptions of identity (a trans woman would be counted in the category of women, etc.). There were no instances of gender non-binary individuals in the reports studied so all personas were reported within two gender categories.

Figure 1 shows summaries for males. The gold bars show the baseline demographic data, the red line shows the pre-intervention values (Fall 2017), and the blue line shows the post-intervention values (Fall 2018).

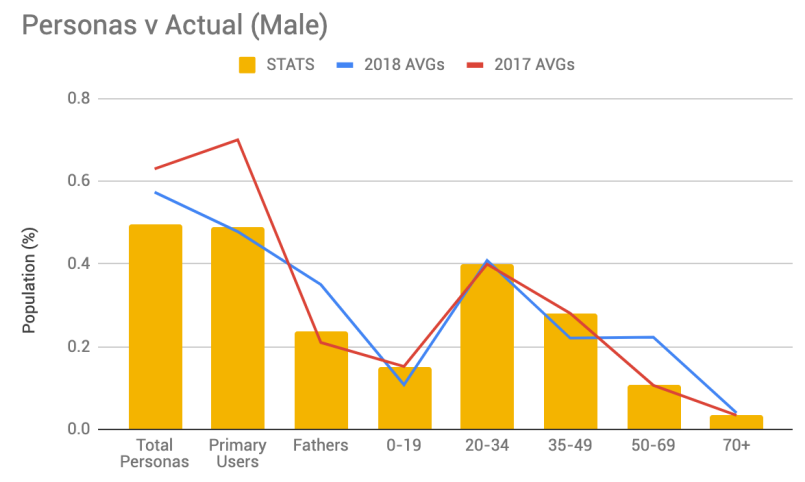

Figure 1: Male personas vs actual demographics.

As is evident from Figure 1, student teams in 2017 over-represented males in their Personas generally but were surprisingly close to baseline for the age distribu- tion. Post-intervention, in 2018, while the general balance of total male Personas was much closer to baseline, there was greater variation with respect to parents and some age groups, especially "middle-aged" men. Furthermore, the percentage of "primary users" allocated to men was much higher in 2017 indicating a previous over-emphasis on males as the primary agents in the design process.

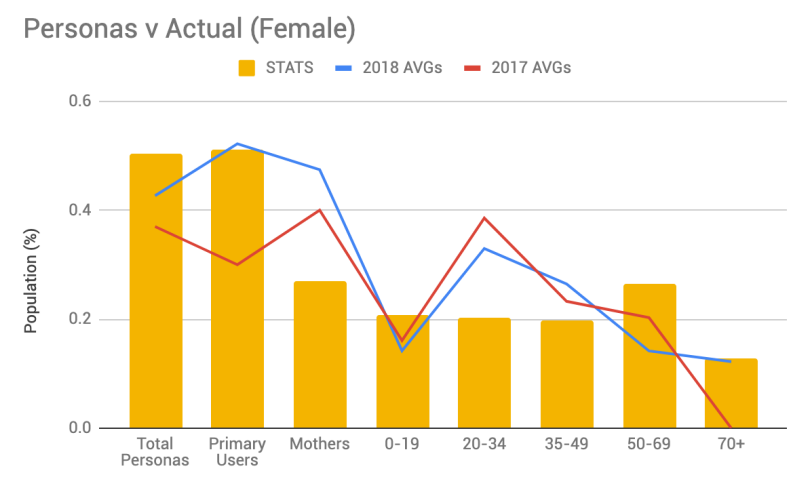

Figure 2: Female personas vs actual demographics.

Figure 2 shows summaries for women. The 2017 data (in red) clearly shows a very skewed distribution of female Personas. Not only were women generally underrepresented, they were overrepresented as mothers, and inaccurately distributed across all age brackets. The 2018 data (in blue) shows a marked improvement compared to 2017 on virtually every measure. Most importantly, elderly females were properly recognized in 2018 whereas they were ignored entirely in 2017 . We still see marked overrepresentation, however, of young adult women and mothers.

Finally, we note that there were several LGBTQ Personas in the 2018 sample, while there was only one in the 2017 sample. We consider this a significant improvement to the diversity of the Persona groups, and a sign that the design focus was broadened to a more reasonable representation of the actual population.

\subsection{Qualitative Analysis}

The qualitative analysis of 2017 reports involved a review of key phrases used in Persona descriptions to determine the human characteristics attributed to those Personas. This analysis was based on the work of Saif Mohammad done for the NRC [2]. In that work, Mohammad identified various descriptors as being either generally positive (e.g., "skilled", "honest") or negative (e.g., "lazy", "neurotic"). For the pre-intervention reports, Nicholson developed a list of 10 positive and 10 negative descriptors based on [2], and counted the occurrence of each descriptor in the Personas of the sample reports, taking care to exclude cases where context indicated a lack of value judgement in the descriptor's use. The descriptors are given in Appendix A.

Nine of the 10 positive descriptors were more common in male Personas than in female Personas, but only three 
of the 10 negative descriptors were more common in males. That is, only one positive descriptor was associated more with women than men, yet seven of 10 negative descriptors were associated more with women than men. In other words, the students were more frequently describing women negatively than men and praising positive characteristics of men more often than women.

In some cases, the differences were quite marked. For instance, $13 \%$ more male than female Personas were identified as "intelligent"; and 25\% more male than female Personas were identified as "mechanically skilled". Conversely, nearly $16 \%$ more female than male Personas were identified as "dependent", "spoiled", or "entitled".

The one positive descriptor that was identified more with women than men was "good social skills", which reflects common stereotypical perceptions of differences between the sexes as described in [3, 4].

In other words, positive descriptors were largely associated with male Personas, and negative descriptors were largely associated with female Personas. This difference has the potential to gender the treatment of Personas throughout the design process, and may reflect a culture of sexism and bias within the classroom. The perceived capabilities or competency of men when compared to women (as captured in these Persona descriptions) are affected by biases and culture as shown in [1].

This qualitative analysis highlighted that many students were using traditional gender roles of superior male intelligence and mechanical skill, with higher female communication skills to describe their user groups. This sex difference bias can affect the design by ignoring variations of abilities within the sexes (by other demographic factors for example), and lumping Personas into two poorly defined groups - where descriptors of "spoiled women" usurped quality, detailed, considerations of disabilities and desires that would impact the design. Furthermore, it was clear in the 2017 reports that very little research was undertaken by the students to investigate diverse characteristics of the actual people who would might be using their design.

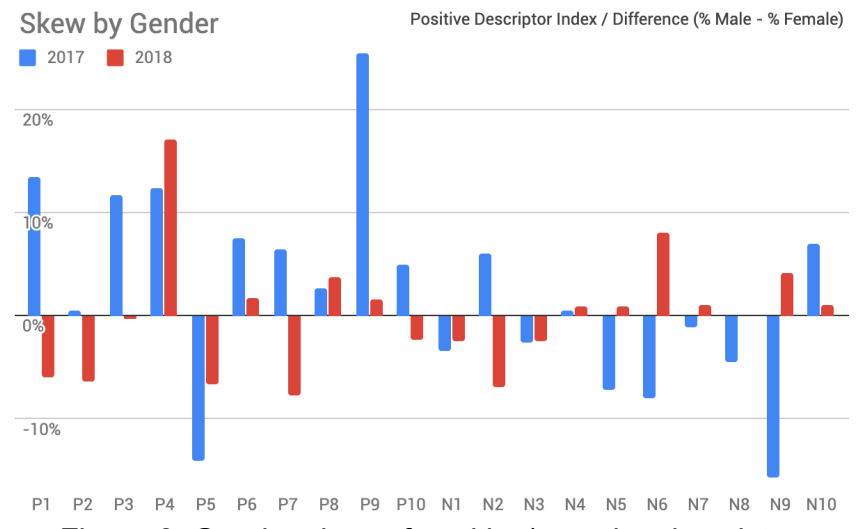

Figure 3: Gender skew of positive/negative descriptors.
Post-intervention, Salustri repeated Nicholson's analysis, searching for the same descriptors in the Persona specifications of the 2018 reports. A summary is shown in Figure 3.

The figure shows the percent difference between male and female Personas exhibiting each of the 10 positive and 10 negative descriptors. Values greater than zero indicate more male than female Personas exhibited a given descriptor; values less than zero indicate the converse. Blue bars show 2017 data; red bars show 2018 data.

The male predominance in positive descriptors in 2017 is evident from the abundance of values greater than zero; similarly, the female predominance in negative descriptors in 2017 is evident from the abundance of values less than zero.

In 2018, not only were positive and negative characteristics more equally distributed between male and female, but also the differences between the number of men versus the number of women exhibiting a given characteristic were smaller.

The positive characteristics of intelligence and strength were far more common in 2018 than any other positive characteristic. This is because there was an explicit requirement to note each Persona's cognitive and physical abilities in the Persona specification chart (which was deployed only in 2018).

An interesting point not evident from Figure 3 involves the negative descriptor of anxiety/depression in 2018, wherein the male:female ratio of occurrence of "anxiety" in Persona descriptions was 1:6, whereas the ratio of occurrence of "depression" was 4:4. That is, "anxiety" was attributed to women six times as often as it was attributed to men; but "depression" was attributed equally to both men and women. It may be that combining these two terms as we did is masking a gender bias. However, further study of this cannot be conducted within this project, and is deferred to future work.

We also found that many descriptors were used in 2018 differently than they were in 2017. In 2017, descriptors like "intelligent", "lazy", "assertive", and "nagging" were simply stated without any context or reference to the design brief. It was common in the pre-intervention reports for the descriptions to seem to express an author opinion, agenda, or desired (traditional gender role) story. There were some examples where the entirety of the persona description appeared intended to demean a woman as being lazy, mean, and demanding - and this was often paired to a male partner persona who was described as hardworking and reasonable. In 2018, on the other hand, while some of these descriptors were used, they were phrased using more neutral language and contextualized to remove negative connotations. For instance, several Personas were noted as "suffering anxiety" (rather than being "highly strung" or "neurotic"); furthermore, underlying causes (such as PTSD due to childhood trauma), were always provided. Due to the compassion and depth 
that was shown by students in describing their Personas with these contexts, we could not arbitrarily ascribe their use of descriptors to merely "positive" or "negative" categories; thus, such cases were excluded.

Relationship Status was explicitly required only in 2018 as part of the Persona specification chart. As a result, it is not surprising that much of this information was missing in 2017 (as indicated by the last row of Table 1, below). Our analysis is thus confined only to 2018 data. Significantly more male than female Personas were married or "in a relationship", and significantly more female than male Personas were single. We are not sure why this is, and will seek to investigate in the future.

Table 1: Summary of Relationship Status data.

\begin{tabular}{|l|c|c|c|c|}
\cline { 2 - 5 } \multicolumn{1}{c|}{} & \multicolumn{2}{c|}{2017} & \multicolumn{2}{c|}{2018} \\
\hline Relationship Status & $\mathbf{M}$ & $\mathbf{F}$ & $\mathbf{M}$ & $\mathbf{F}$ \\
\hline married & $17 \%$ & $26 \%$ & $48 \%$ & $36 \%$ \\
\hline single & $13 \%$ & $16 \%$ & $33 \%$ & $41 \%$ \\
\hline divorced/widowed & $8 \%$ & $11 \%$ & $8 \%$ & $8 \%$ \\
\hline "in a relationship"/engaged & $4 \%$ & $5 \%$ & $10 \%$ & $6 \%$ \\
\hline n/a, "it's complicated", other & $58 \%$ & $37 \%$ & $0 \%$ & $0 \%$ \\
\hline
\end{tabular}

Occupation information could not be captured reliably from the 2017 reports because Personas were described in free-format text. Nonetheless, the 2018 results, summarized in Table 2, suggest a possible gender bias: relatively few women held blue-collar jobs, and no men were "stay at home" spouses.

Table 2: Summary of Occupation data.

\begin{tabular}{|l|c|c|}
\hline Occupation & \multicolumn{2}{c|}{2018} \\
\hline white collar & M & F \\
\hline blue collar & $42 \%$ & $46 \%$ \\
\hline retired & $49 \%$ & $25 \%$ \\
\hline stay at home & $3 \%$ & $10 \%$ \\
\hline unemployed & $0 \%$ & $4 \%$ \\
\hline gamer/unconventional/other & $6 \%$ & $7 \%$ \\
\hline
\end{tabular}

Finally, information about Education could not be captured reliably from the 2017 reports for similar reasons. The 2018 data is summarized in Table 3.

Table 3: Summary of Education data.

\begin{tabular}{|l|c|c|c|c|}
\cline { 2 - 5 } \multicolumn{1}{c|}{} & \multicolumn{2}{c|}{2018} & \multicolumn{2}{c|}{ Cdn. Pop. } \\
\hline Education & M & F & M & F \\
\hline$<$ high school & $9 \%$ & $12 \%$ & $19 \%$ & $18 \%$ \\
\hline high school & $35 \%$ & $19 \%$ & $26 \%$ & $27 \%$ \\
\hline trade / college & $24 \%$ & $12 \%$ & $33 \%$ & $31 \%$ \\
\hline college / university & $30 \%$ & $36 \%$ & \multirow{2}{*}{$22 \%$} & \multirow{2}{*}{$25 \%$} \\
\hline$>1$ university degree & $9 \%$ & $16 \%$ & & \\
\hline
\end{tabular}

Table 3 shows a distinct gender bias compared to Statistics Canada data. The actual population enjoys surprisingly similar proportions between men and women: the differences for all levels of education are only a few per- cent. However, the 2018 Personas show marked differences: far more male than female Personas had schooling up to and including trade schools / technical college, but far more female than male Personas had College or University education. The reason for this disparity is not clear and remains a question for future study.

\subsection{Exit Survey Analysis}

An exit survey was distributed at the end of the semester to all students. Responses were anonymous. Of the 282 students in the 2018 class, we received 32 responses. Summaries of responses are provided in Appendix B. The questions were intended to ascertain how students viewed the new gender and diversity material. The results were overwhelmingly positive: over $87 \%$ of respondents agreed or strongly agreed with statements describing the new material having various positive effects.

Most significantly, $87.6 \%$ of respondents agreed or strongly agreed with the statement "I feel supported and respected in my program of study." We believe this is an important step to improve the inclusion, and well-being of our students and the cohesiveness of our academic community.

\section{DISCUSSION}

Generally, we believe our interventions had a positive effect on students. Demographic distribution of Personas improved in 2018; LGBTQ Personas were included and given respect and agency equal to other Personas; the incidence of superficial use of both positive and negative personality descriptors decreased radically in 2018, suggesting a deeper and more compassionate view of users generally. The exit survey was overwhelmingly positive, leading us to believe we are on the right track.

The design brief for all teams sampled in both 2017 and 2018 was to "develop a way to change automobile tires by the roadside." This could have influenced the distribution of Personas away from Statistics Canada baseline. Several teams in both years found by way of background research that most tires are changed by males. At least one team reasoned that women "cannot" change tires. Fortunately, TAs and instructors were able to intervene and lead them to recognize the flaw in their reasoning before their final reports were submitted. It would have been interesting to try to tabulate all similar occurrences in all the sample teams during the semester, but we lacked the resources to do this. More broadly, the issue of biases induced in students as a result of performing necessary background research remains an open question that points to broader social and cultural opinions on gender roles.

The proportion of women in the student teams that we sampled was approximately $20 \%$ in both 2017 and 2018, with some teams having no women members and others have as many as $50 \%$ women. The effect of the diversity 
of the team members on the diversity of the Personas was not clear enough to draw conclusions.

We did not have the resources to study the sections of the class that were assigned the Baby Stroller design brief. Anecdotally, the TAs and instructors noted no particular variations in the performance of those teams, or in the quality of their final designs, compared to other sections. However, there is no way to be sure without detailed study of the reports themselves. Fortunately, those reports remain on file and we hope to be able to review them carefully in the future.

Some apparent biases remain, especially with respect to proportional representation of women with respect to relationship status, occupation, and education. Also, there are yet more complex intersections of identity not studied here (race, socioeconomic status, etc.) which may still be limiting the inclusiveness of student designs. Future work will further explore the ways biases may be influencing student work in this course, and additionally intervene to increase student ability to design for a diverse world.

\section{CONCLUSIONS}

Utilizing Human Factors in engineering design process improves the quality and usability of products by focusing on the needs, goals, and abilities of Personas in user groups. However, this process is limited by the biases of the designer, as was seen in student reports which were studied for their descriptions and handling of women and women's issues in the term project report. Therefore, an intervention was created which modified the course to explicitly guide students to a more diverse and equitable approach to the creation and use of their user groups. Overall, this intervention resulted in better representation of the demographics of the population, more mature and respectful communication of Persona characteristics, and a course culture of support and inclusion as seen in the student survey responses.

Due to Ryerson Policies, we cannot link directly to the courseware that was developed in this project. However, we are willing to provide those materials to anyone requesting them for teaching purposes; contact the corresponding author by email to obtain a copy.

\section{Acknowledgements}

The authors acknowledge the financial support of the Learning and Teaching Office at Ryerson University.

\section{References}

[1] Jon A. Leydens, Juan C. Lucena, Engineering Justice: Transforming Engineering Education and Practice, Piscataway, IEEE Press, 2017.

[2] S. Mohammad, "NRC Word-Emotion Association Lexicon", Saifmohammad.com, Available as of 18 April 2019 from
http://saifmohammad.com/WebPages/NRC-EmotionLexicon.htm.

[3] J T Richardson, P J Caplan, M Crawford, J S Hyde, and J T E Richardson, Gender Differences in Human Cognition, New York, Oxford University Press, 1997.

[4] A. Saini, Inferior: How science got women wrong and the new research that's rewriting the story, Boston, Beacon Press, 2017.

[5] F.A. Salustri, \& W.P. Neumann. "Creating a HumanCentric Engineering Design Course," in Proc. 2017 Conference of the Canadian Engineering Education Association. (Toronto, ON; 4-7 June 2017), 7 pp, 2017. Available as of 18 April 2019 from http://digital.library.ryerson.ca/islandora/object/RULA \%3A5576.

[6] F. A. Salustri and W.P. Neumann. "Combining Hierarchical Task Analysis and Usage Scenarios to Help Embed Human Factors in Design," In Proc. 2018 Conference of the Canadian Engineering Education Association. (Vancouver, BC; 3-6 June 2018), 7 pp, 2018. Available as of 19 April 2019 from https://drive.google.com/file/d/1Og3kQ3V00LO3dVfj ez-iNJagN1cviTPS/view.

\section{APPENDIX A: PERSONA DESCRIPTORS}

The 10 positive Persona descriptors used to analyze student reports were:

1. Intelligent, Very Smart, Smart, Competent, fast learner, quick to react

2. Patient, steady, level-headed

3. committed, good at job, dedicated, passionate

4. Strong, perfectly healthy, healthy, fit

5. good social skills, kind, positive, easy going, good leader

6. Routine oriented, perfectionist, organized

7. independent, ambitious

8. careful with money

9. mechanically skilled, technologically skilled, mechanic, handyman

10. confident, courageous

The 10 negative Persona descriptors used were:

1. Stressed, Nervous, worry-wart

2. Anxiety, depression

3. Weak

4. lazy, late

5. stubborn, rebellious

6. Unprepared, last minute, rushed, distracted, careless, impatient, hurried

7. Bad driver

8. nags

9. dependent, entitled, spoiled, demanding, short tempered, no filter in comm. 
10. mechanically challenged, technologically challenged

\section{APPENDIX B: QUALITY ASSURANCE}

The following charts summarize the 32 responses to the exit survey administered voluntarily and anonymously to students in 2018.

Having a focus on diversity, equity, and inclusion will improve a designer/engineer's ability to develop a good design. 32 responses

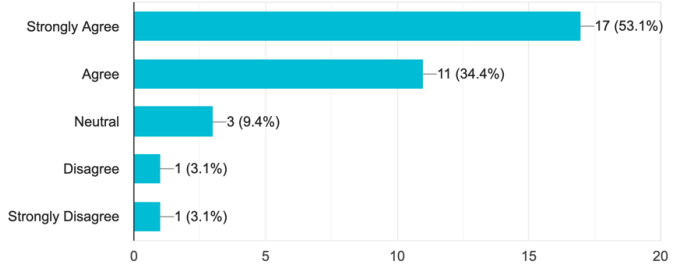

I understand what it means to be inclusive in design. 32 responses

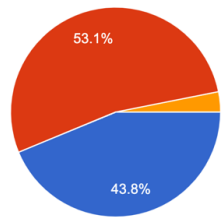

- Strongly agree

- Agree

Disore

- Strongly disagree

I feel supported and respected in my program of study. 32 responses

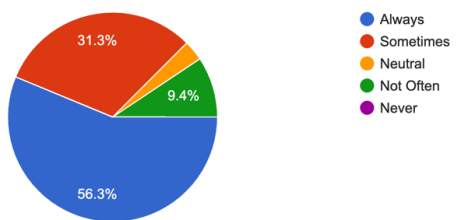

When designing a product, it is important for an engineer/designer to research and understand many different groups in society. 32 responses

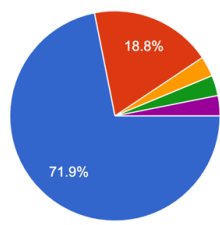

- Strongly Agree

Agree

- Disagree

- Strongly Disagree

What is/are the best way(s) to design for women? 32 responses

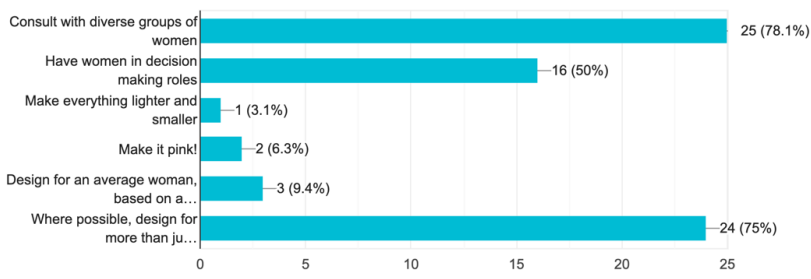

I found these slideshows useful:
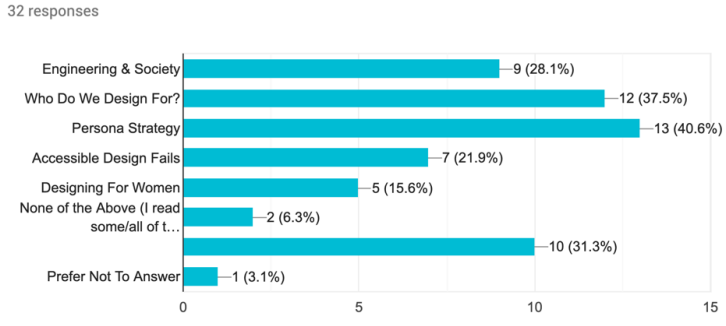